\title{
Wideband MIMO antenna for SCADA Wireless Communication Backhaul Application
}

\author{
Nur Zafirah Bt Muhammad Zubira, Razali Ngah ${ }^{\mathrm{b}}$, Vincent Yong Kai Loung ${ }^{\mathrm{c}}$, Reza Firsandaya Malik ${ }^{\mathrm{d}}$, \\ a ,b, c Wireless Communication Centre, School of Electrical Engineering, Faculty of Engineering, Universiti Teknologi Malaysia \\ 81310 Skudai, Johor Bahru, Johor, Malaysia \\ ${ }^{\mathrm{d}}$ Faculty of Computer Science, Universitas Sriwijaya, Palembang, 30128, Indonesia
}

Article History: Received: 10 November 2020; Revised 12 January 2021 Accepted: 27 January 2021; Published online: 5 April 2021

\begin{abstract}
A wideband multiple-input-multiple-output (MIMO) antenna system with common elements suitable for SCADA wireless communication backhaul application which is operating frequency of $0.85-2.6 \mathrm{GHz}$ that can cover global system for mobile communication (GSM) $900 \mathrm{MHz}$ and $1.8 \mathrm{GHz}$, The Universal Mobile Telecommunication System (UMTS) 2GHz, WiFi $(2.4 \mathrm{GHz})$ and Long Term Evolution (LTE) $2.6 \mathrm{GHz}$ is proposed. The proposed MIMO antenna system consists of four microstrip feedline with common radiating element and a frame shaped ground plane. A single port antenna also was designed and presented in this paper to show the process to design wideband MIMO antenna structure. The radiator of the MIMO antenna system is designed as the shape of modified rectangle with straight line at each corner to enhance the bandwidth frequency. To improve the isolation between ports, the ground plane is modified by inserting four L-slots in each corner to reduce mutual coupling. For an antenna efficiency of more than $60 \%$, the simulated reflection coefficients are below $-10 \mathrm{~dB}$ for all ports at expected frequency. Simulated isolation is achieved greater than $-10 \mathrm{~dB}$ by using a modified ground plane. Also, a low envelope correlation coefficient (ECC) less than 0.1 and polarization diversity gain of about 10dB with the orthogonal mode of linear polarization and omnidirectional pattern during the analysis of the radiation characteristic are achieved. Therefore, the proposed design can be used for SCADA wireless communication backhaul application..

Keywords: Diversity gain, envelope correlation coefficient (ECC), ground plane, isolation, Global System for Mobile communication (GSM), Long Term Evolution (LTE), Multiple-Input-Multiple-Output (MIMO)
\end{abstract}

\section{Introduction}

Supervisory Control and Data Acquisition (SCADA) systems are used in industrial and engineering applications to monitor and control distributed systems from master location. SCADA communication is intended to provide the means by which data can be transferred between master stations and Remote Terminal Units (RTUs). RTUs communicate with master station using few types of communication via Remote Front End (RFE) [1]. One of the types of the communication was used at secondary stations is General Packet Radio Service (GPRS) wireless communication. GPRS is a packet oriented mobile data standard on $2 \mathrm{G}$ and $3 \mathrm{G}$ cellular communication network's global system for mobile communication (GSM) and The Universal Mobile Telecommunication System (UMTS) respectively. However, this communication needs to be improved up to 4G/LTE since there always have intermittent wireless communication link between RTUs and master station. But at certain locations of secondary stations, the coverage network of 4G/LTE was not supported. Therefore, the proposed MIMO antenna system need to cover the frequency range up to LTE frequency band. There are currently six spectrum bands, $850 \mathrm{MHz}$, $900 \mathrm{MHz}, 1800 \mathrm{MHz}, 2100 \mathrm{MHz}, 2300 \mathrm{MHz}$ and $2300 \mathrm{MHz}$ bands which have been assigned or allowed to be used by operator to provide mobile broadband service in Malaysia [5].

Multiple Input Multiple Output (MIMO) is one of the major technological breakthroughs in 1990s in the field of signal processing. To transmit several independent data streams at longer range on the same time or frequency using spatial multiplexing, it relies on the use of the multiple antennas at the transmitter and the receiver [2]. MIMO wireless technology has been used by Wi-Fi, LTE and many other radio, wireless and RF technologies because it can provide increased link capacity and spectral efficiency combined with improved link reliability. However, the antenna tends to be strong mutual coupling leads to high correlation and resulting the performance parameters of MIMO antenna are attenuated due to it closely placed antenna elements. Several methods have been investigated to reduce mutual coupling in a MIMO antenna system with closely space elements. Some commonly techniques are by using parasitic element, electromagnetic band-gap (EBG) structures, decoupling and matching networks and neutralizing line [3].

In this paper, a wideband MIMO microstrip patch antenna operating at the frequency band of $0.85-2.6 \mathrm{GHz}$ is presented. The operating frequency covers the frequency range that suitable for GSM $(880 \mathrm{MHz}$ and $1.8 \mathrm{GHz})$, UMTS $(2 \mathrm{GHz}), \mathrm{WiFi}(2.4 \mathrm{GHz})$ and LTE $(2.6 \mathrm{GHz})$ applications. In addition, a wideband single port antenna that 
cover the operating frequency also presented in order to achieve four port wideband MIMO antenna. The proposed MIMO antenna system consists symmetrical four port microstrip feedline, straight line on monopole patch antenna with single radiating edges and a frame shaped ground on ground plane. The straight line on monopole patch excited resonant frequency over the bandwidth and increased as long as the bandwidth is covered. The frame shaped ground with L-slots at the corner reduce the effect of coupling among the ports of microstrip feed line which leads to improvement on the performance of the MIMO antenna in terms of its reflection coefficients, isolation, radiation characteristics and MIMO performance. In Section 2, the configuration of antenna process of the presented antenna is described in detail. The simulation results including results for single port antenna, optimization of wideband MIMO antenna and the proposed wideband MIMO antenna are discussed in Section 3.

\section{Antenna Configuration}

\section{A. Single Port antenna}

In the process of designing wideband MIMO antenna, single port antenna is designed as a first step for next improvement. Fig. 1 shows demonstration of the designed single port antenna. This designed antenna consists of monopole patch antenna with single radiating element and a microstrip feedline and ground. The electromagnetic software CST is used to simulate the designed antenna. FR-4 dielectric substrate with relative permittivity $\varepsilon_{\mathrm{r}}=4.3$, thickness $\mathrm{h}=1.6 \mathrm{~mm}$, substrate dimensions of length $\mathrm{L}_{\mathrm{s}}$ and width $\mathrm{W}_{\mathrm{s}}$ are used. Radiating patch and ground are made of copper material with thickness $t=0.035 \mathrm{~mm}$ and conductivity $\sigma=5.96 \mathrm{e}^{7} \mathrm{~s} / \mathrm{m}$. The ground plane width same as $\mathrm{W}_{\mathrm{s}}$ while the length $\mathrm{L}_{\mathrm{g}}=18 \mathrm{~mm}$ and remain constant. To achieve $50 \Omega$ output impedance matching with SMA connector, a transmission line fed with width $\mathrm{W}_{\mathrm{f}}=3 \mathrm{~mm}$ and length $\mathrm{L}_{\mathrm{f}}=20 \mathrm{~mm}$ is used.

In designing the expected antenna with required operating frequency, the step cut at each corner using the Stepped Cut at Four Corners (SCFC) method has been used. This method usually used to design multi-mode or broadband modified rectangular microstrip patch antennas [4]. By increasing the number of steps at the corners, the number of excited resonance frequencies over the bandwidth are increasing. As shown in Fig.1, the ten stepped cut is created at the corners of the main patch for ensuring coverage frequencies by the antenna. $W_{1}$ and $L_{1}$ indicate width and length of the step. The dimension of the main patch is obtained at the lowest frequency $(850 \mathrm{MHz})$.

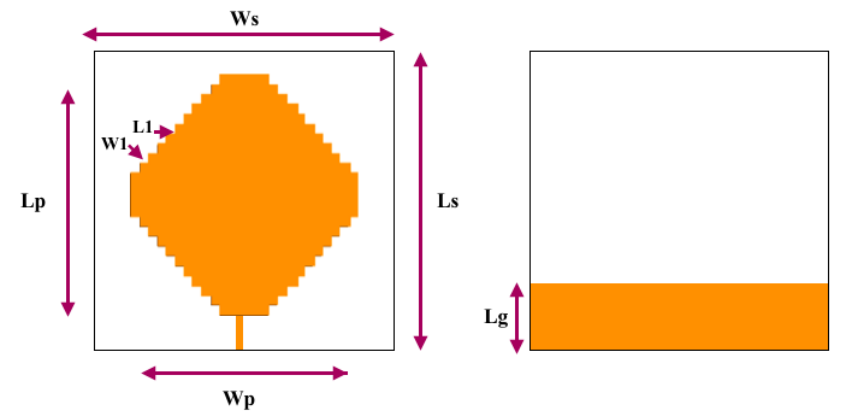

Figure 1: Geometry of single port antenna (front and back)

Table 1: Dimension of single port antenna

\begin{tabular}{|l|l|l|l|l|l|l|}
\hline Parameter & $\mathbf{W}_{\mathbf{s}}$ & $\mathbf{L}_{\mathbf{s}}$ & $\mathbf{W}_{\mathbf{p}}$ & $\mathbf{L}_{\mathbf{p}}$ & $\mathbf{W}_{\mathbf{1}}$ & $\mathbf{L}_{\mathbf{1}}$ \\
\hline $\begin{array}{l}\text { Value } \\
(\mathbf{m m})\end{array}$ & 90 & 140 & 84.9 & 108.4 & 2.87 & 3.63 \\
\hline
\end{tabular}

\section{B. MIMO wideband antenna}

Wideband MIMO antenna is designed after some improvements have been made. A port is added one by one on the single port antenna in order to designed MIMO antenna. However, the coverage of bandwidth by the MIMO antenna was not achieved if the dimensions as in Table 1 are remained. Therefore, the improvement that have been made are increasing the size of patch and substrate in order to achieve the required operating frequency. If the number of steps at the corners increases infinitely, the steps become smaller and reach a point that the step path $\left(\mathrm{W}_{1}\right.$ and $\mathrm{L}_{1}$ ) acts as a line (a) with infinite points. Besides, a frame shaped ground is inserted a L-slots at each corner in order to reducing the effect of coupling among the ports of microstrip feed line which leads to improvement on the performance of the MIMO antenna. The geometry of MIMO antenna is shown in Fig 2. 


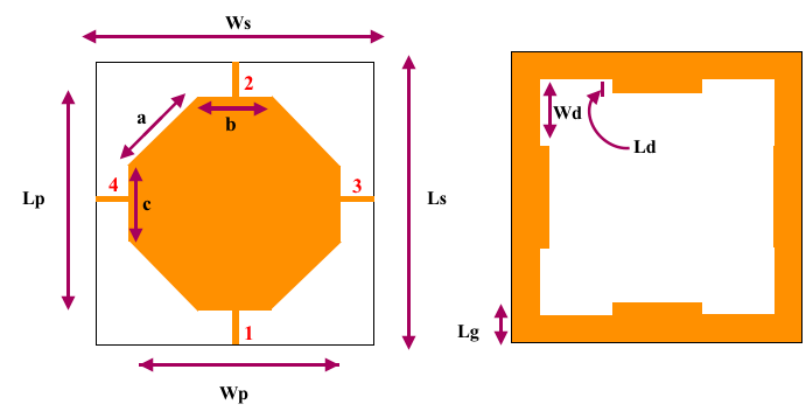

Figure 2: Geometry of MIMO antenna (front and back)

Table 2: Dimension of MIMO antenna

\begin{tabular}{|l|l|l|l|l|l|l|l|}
\hline Parameter & $\mathbf{W}_{\mathbf{s}}$ & $\mathbf{L}_{\mathbf{s}}$ & $\mathbf{W}_{\mathbf{p}}$ & $\mathbf{L}_{\mathbf{p}}$ & $\mathbf{a}$ & $\mathbf{b}$ & $\mathbf{c}$ \\
\hline $\begin{array}{l}\text { Value } \\
(\mathbf{m m})\end{array}$ & 270 & 96.3 & 230 & 206.6 & 132.3 & 35.2 & 27.5 \\
\hline
\end{tabular}

\section{Simulation Results And Discussion}

\section{A. Single port Antenna}

As shown in Table 1, dimensions of patches are being simulated with five and ten stepped cut. The comparison between both designs are shown in Figure 3. Based on the result, it can be seen that with ten stepped cut at the corners of the main patch can cover frequency between $850 \mathrm{MHz}$ to $2.6 \mathrm{GHz}$ compared to five stepped cut. If with five stepped cut, the range frequency from 1.8 to $2.1 \mathrm{GHz}$ is not covered. Therefore, the number of step is increased to ten and wideband single port antenna is obtained at desired frequency range to cover frequency of GSM (880MHz and 1.8GHz), UMTS (2GHz), WiFi $(2.4 \mathrm{GHz})$ and LTE $(2.6 \mathrm{GHz})$.

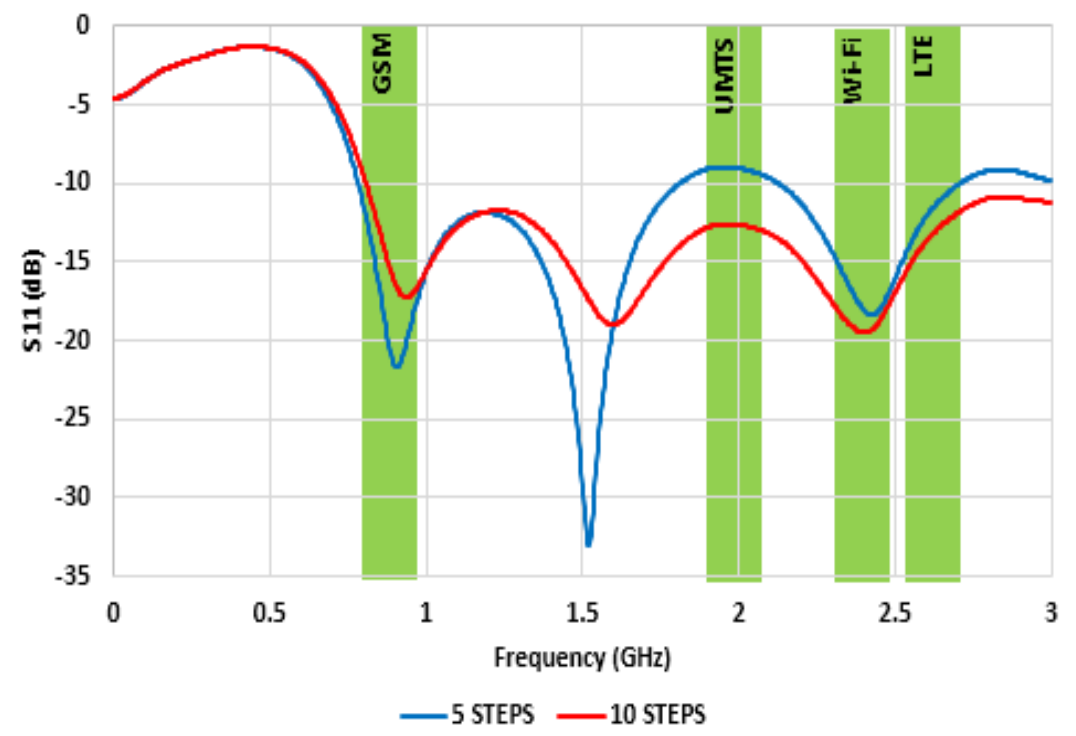

Figure 3: Return loss of single port antenna $(\mathrm{dB})$

Stable omnidirectional at $900 \mathrm{MHz}$ and approximate bidirectional at the upper frequency have been achieved for the far field radiation pattern of proposed single port antenna over the bandwidth. Figure 4 shows the simulated normalized $\mathrm{E}$ and $\mathrm{H}$-plane radiation patterns of proposed antenna for frequency at $900 \mathrm{MHz}$ and $2.6 \mathrm{GHz}$. 


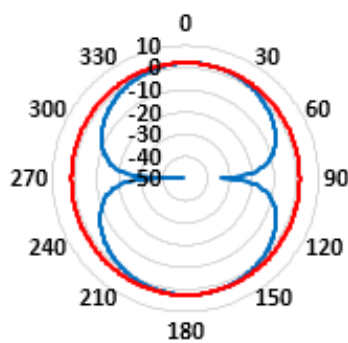

-E-PLANE - H-PLANE

(a)

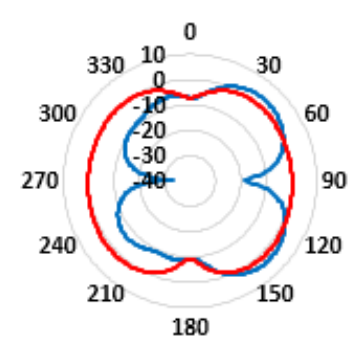

-E-PLANE - H-PLANE

(b)

Figure 4: Radiation pattern of single port antenna at frequency (a) $900 \mathrm{MHz}$, (b) $2.6 \mathrm{GHz}$

\section{B. Optimization of Wideband MIMO antenna}

In getting the geometry of MIMO antenna as shown in Fig.2, some optimizations have been made by adding one by one port to the single port antenna. The dimensions of main patch for MIMO antenna is listed in Table 2 are different with Table 1. The MIMO antennas are simulated with stepped cut at each corner of the main patch with different dimensions of main patch and frame shaped ground without slots. From Fig.5, it can be seen that the S11 is improved when the dimensions of main patch bigger compared to dimensions of patch in Table 1. This is because the bigger the size of the patch, the lower frequency it can be covered by the antenna. Therefore, the dimensions of patch as in Table 2 are chosen.

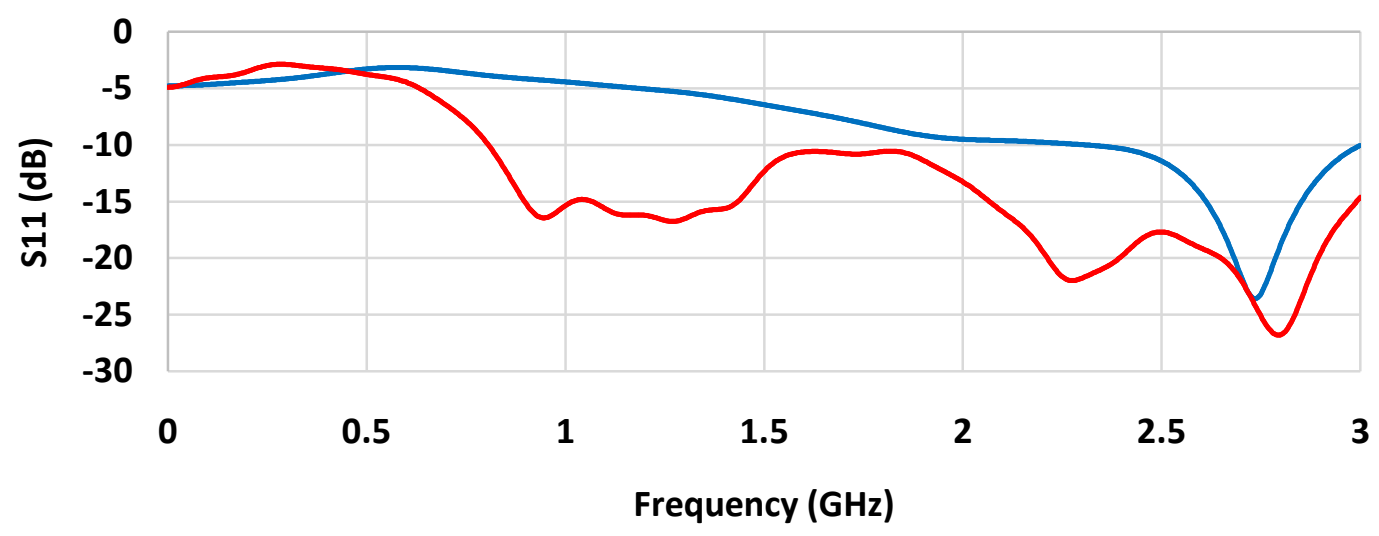

_Dimensions of patch as in Table 1 Dimensions of patch as in Table 2

Figure 5: S11 for MIMO antenna with straight lines at the corners with different dimensions of patch

Then, MIMO antenna with dimensions of patch in Table 2 with frame shaped ground without slots are simulated with stepped cut and straight line at the corners of the patch. The comparisons of S11 for both designs shown as in Fig. 6. The result shows that when stepped cut is replaced with straight line, the frequency bandwidth is improved. Therefore, the straight lines at each corner is fomed. 


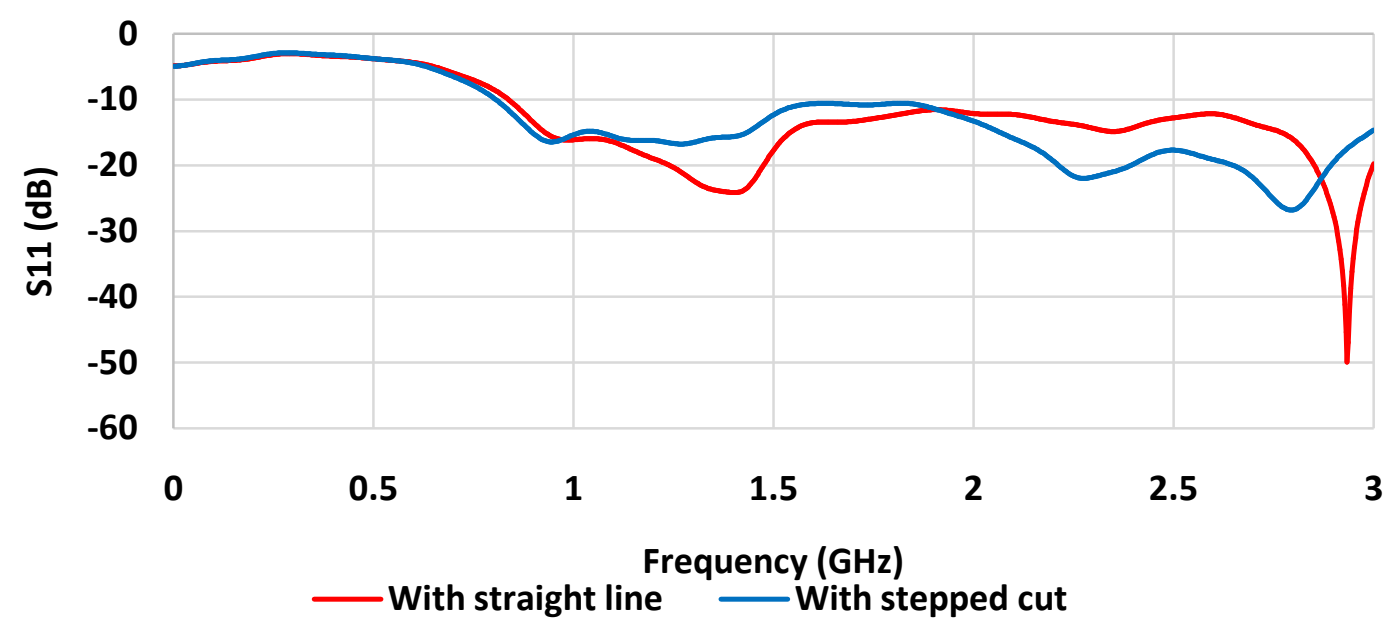

Figure 6: S11 for MIMO antenna with straight lines and stepped cut at each corners

To enhance the isolation between the ports, a technique to decrease coupling between adjacent antennas is implemented. Since the structure of the ports are in perpendicular, the simplest method that can be made is to move them farther apart and orient them so that their polarizations are mismatched. The ground plane is modified by introducing four slots. Fig. 2 shows the ground plane slots, which are created at the four corners with dimensions of width $\mathrm{W}_{\mathrm{d}}=65 \mathrm{~mm}$ and length $\mathrm{L}_{\mathrm{d}}=10 \mathrm{~mm}$. The current distribution of the proposed MIMO antenna without slots in ground plane clarified the main current flows via stepped lines of the radiator and the L-corner ground plane. From Fig.7, it is found that by creating the slots in the corners of the ground plane, the current coupling across the Lcorner of the ground plane is decreased. A variety of results are obtained by changing the dimensions of the slots. Therefore, to show the effect of the slot dimensions on isolation between ports, the parametric study on $\mathrm{L}_{\mathrm{d}}$ and $\mathrm{W}_{\mathrm{d}}$ are shown in Fig. 8 and Fig. 9 respectively. It shows how mutual coupling is affected by varying the $\mathrm{L}_{d}$ from $1 \mathrm{~mm}$ to $10 \mathrm{~mm}$, and $\mathrm{W}_{\mathrm{d}}$ from $50 \mathrm{~mm}$ to $100 \mathrm{~mm}$. It can be seen that at $0.9 \mathrm{GHz}, \mathrm{S} 31$ in Fig. 8 is changed from $-13.3 \mathrm{db}$ to $19.7 \mathrm{~dB}$ while in Fig.9 does not have much changes which is from $-13.3 \mathrm{db}$ to $-12.8 \mathrm{~dB}$.

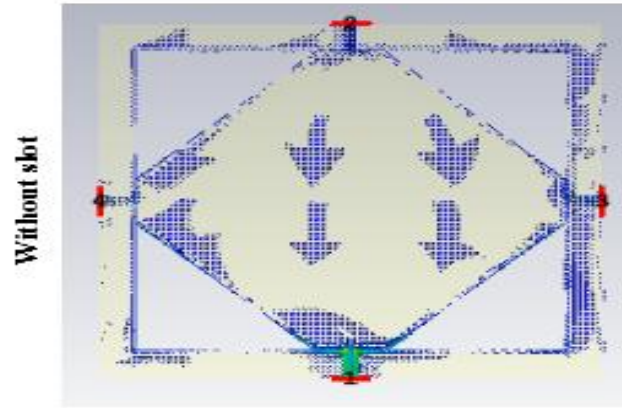

(a)

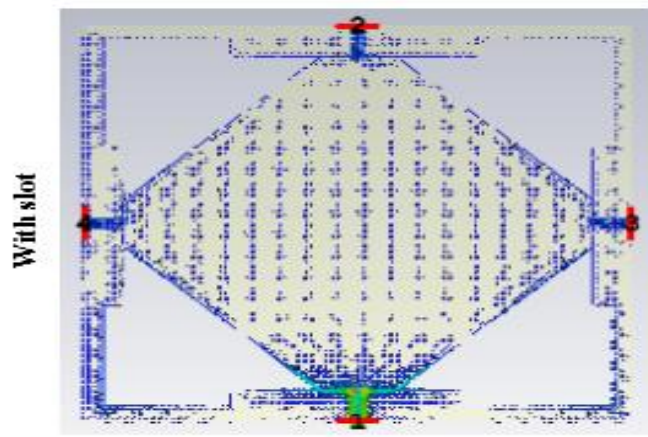

(c)

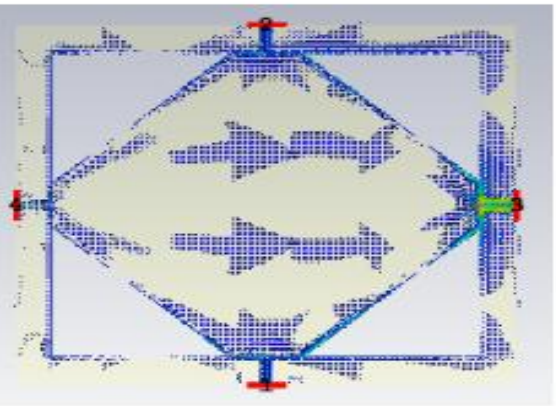

(b)

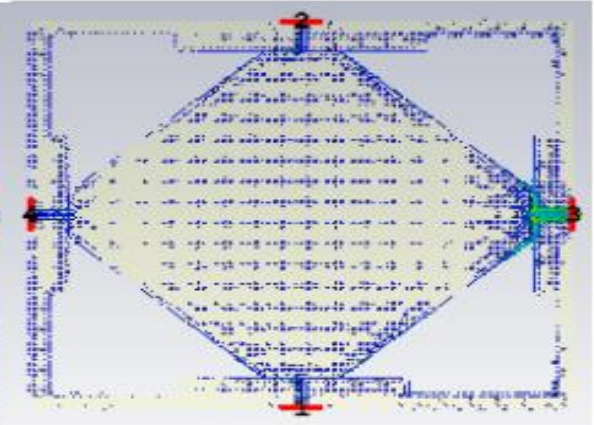

(d)

Figure 7: Current distributions of the MIMO antenna with and without slot at 0.9GHz. (a) Port 1 excited. (b) Port 3 excited. (c) Port 1 excited (d) Port 3 excited. 


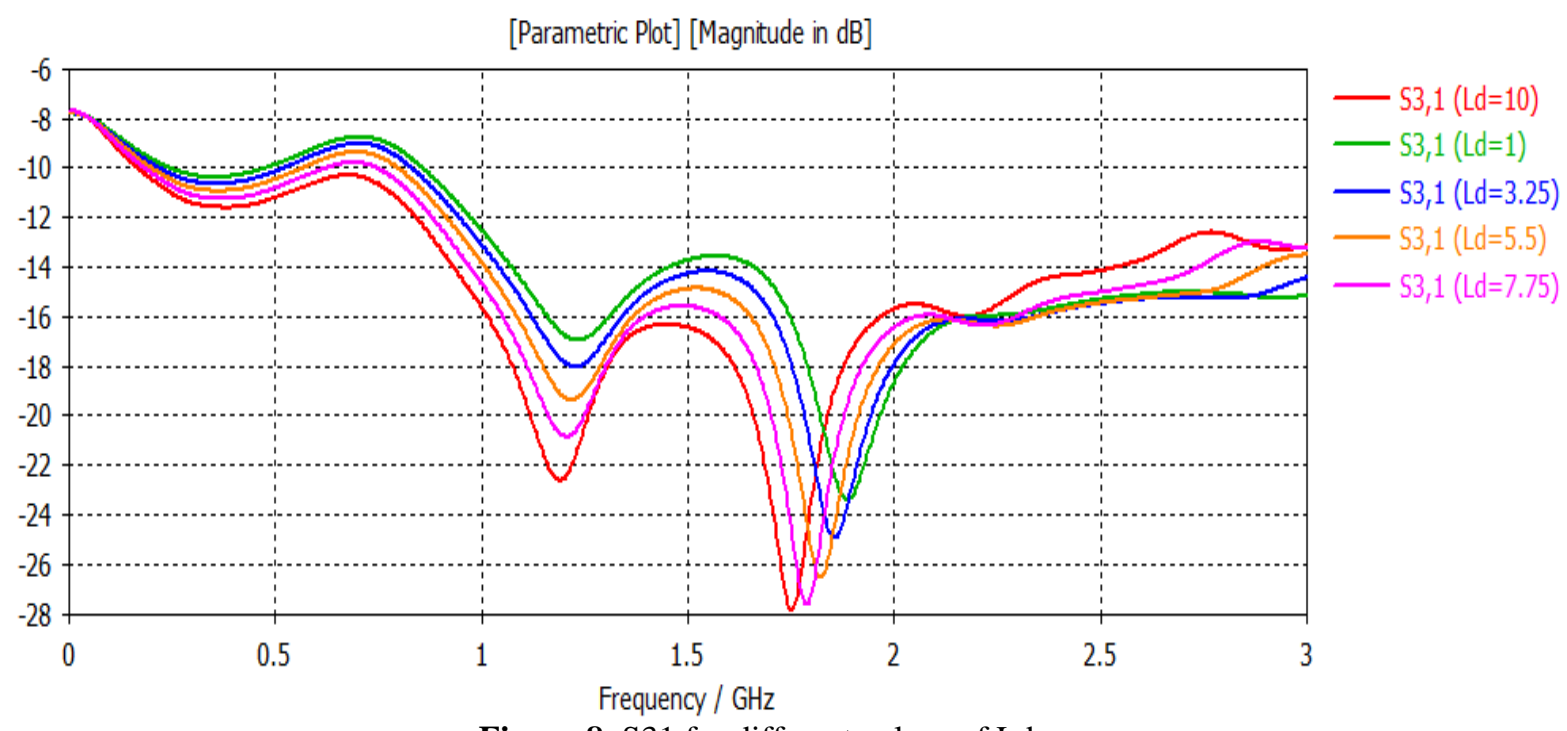

Figure 8: S31 for different values of $\mathrm{Ld}$

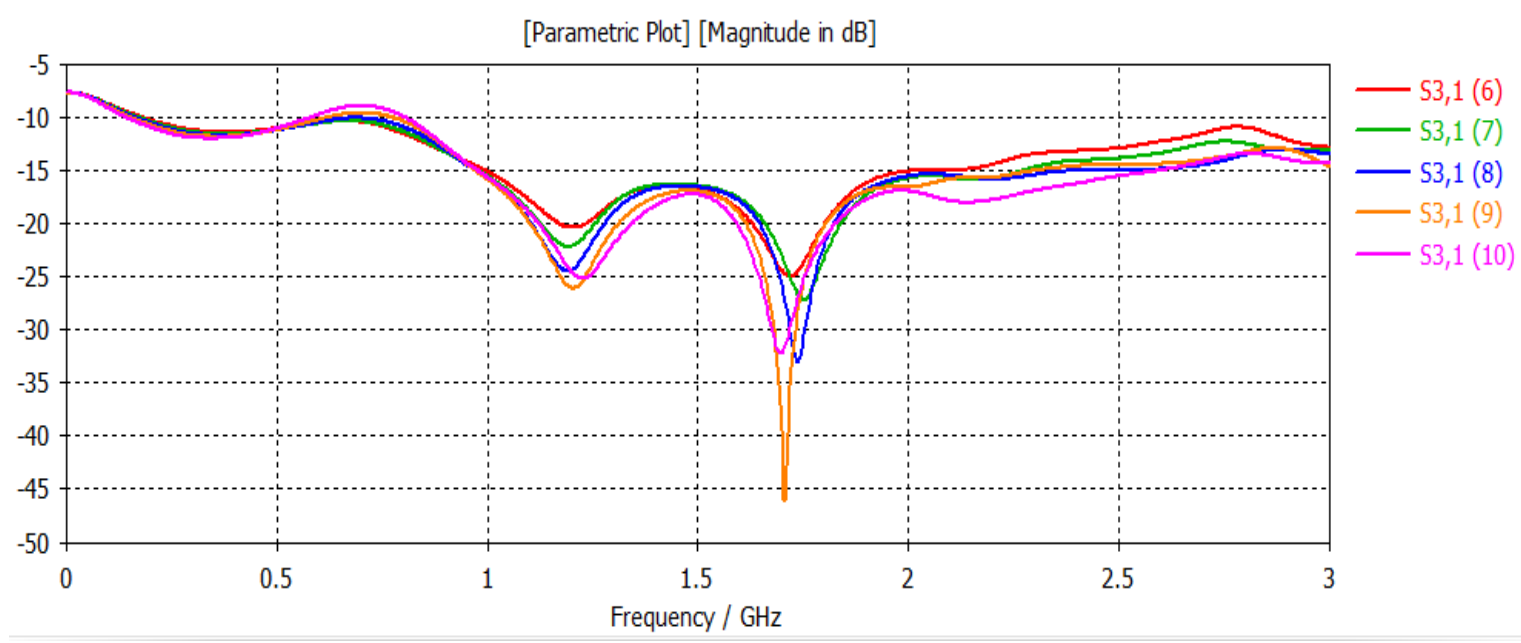

Figure 9 : S31 for different values of Wd

\section{The proposed wideband MIMO antenna}

The simulated reflection coefficients of the proposed MIMO antenna system are shown in Fig.10. It is observed that all S-parameter can achieve the desired frequency range. Therefore, the proposed MIMO antenna can be used to cover the desired range frequency of $0.85-2.6 \mathrm{GHz}$ which is to support GSM, UMTS Wi-Fi and LTE frequencies. 


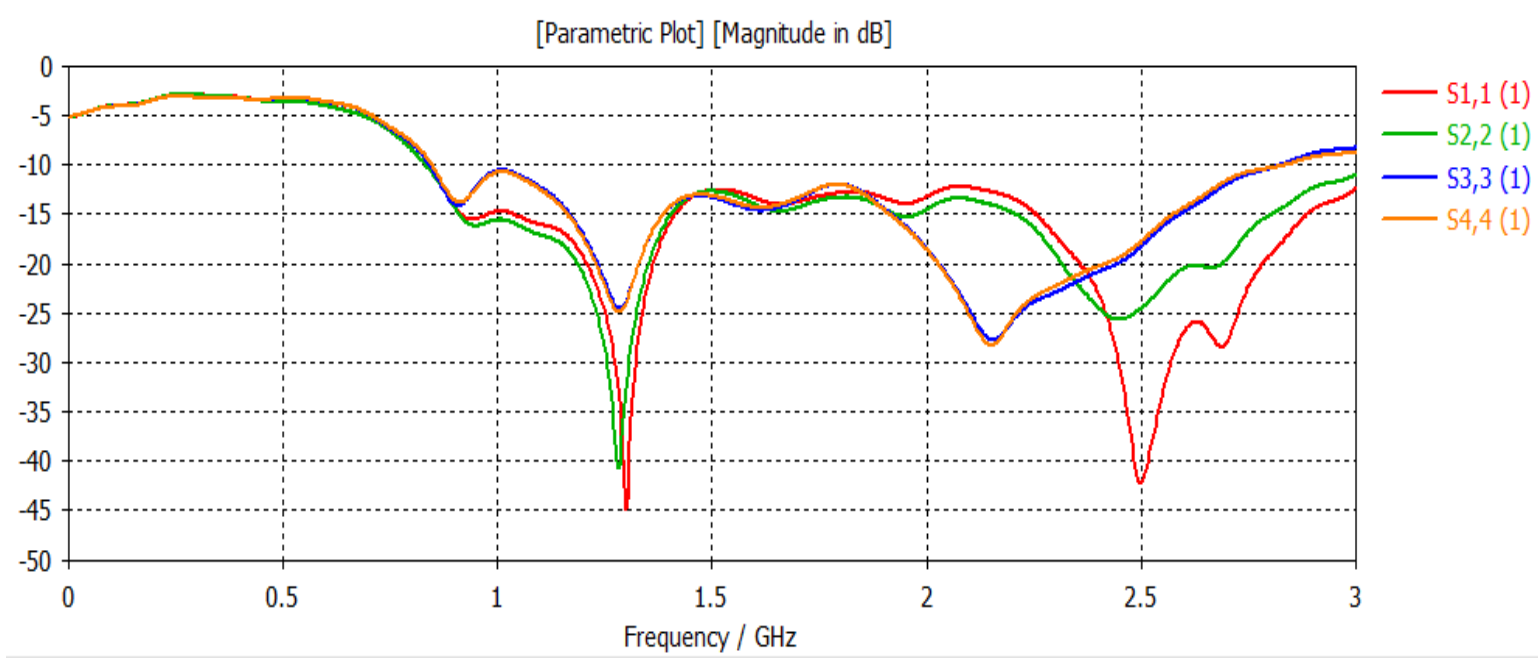

Figure 10: Simulated reflection coefficients of MIMO antenna system

The simulated isolation between antennas is represents in Fig.11. Each curve represents the isolation between two ports. The results are in good performance for MIMO applications from 0.85 to $2.6 \mathrm{GHz}$ with isolation greater than $-10 \mathrm{~dB}$. Hence, it is an acceptable isolation between the ports in the MIMO antenna system.

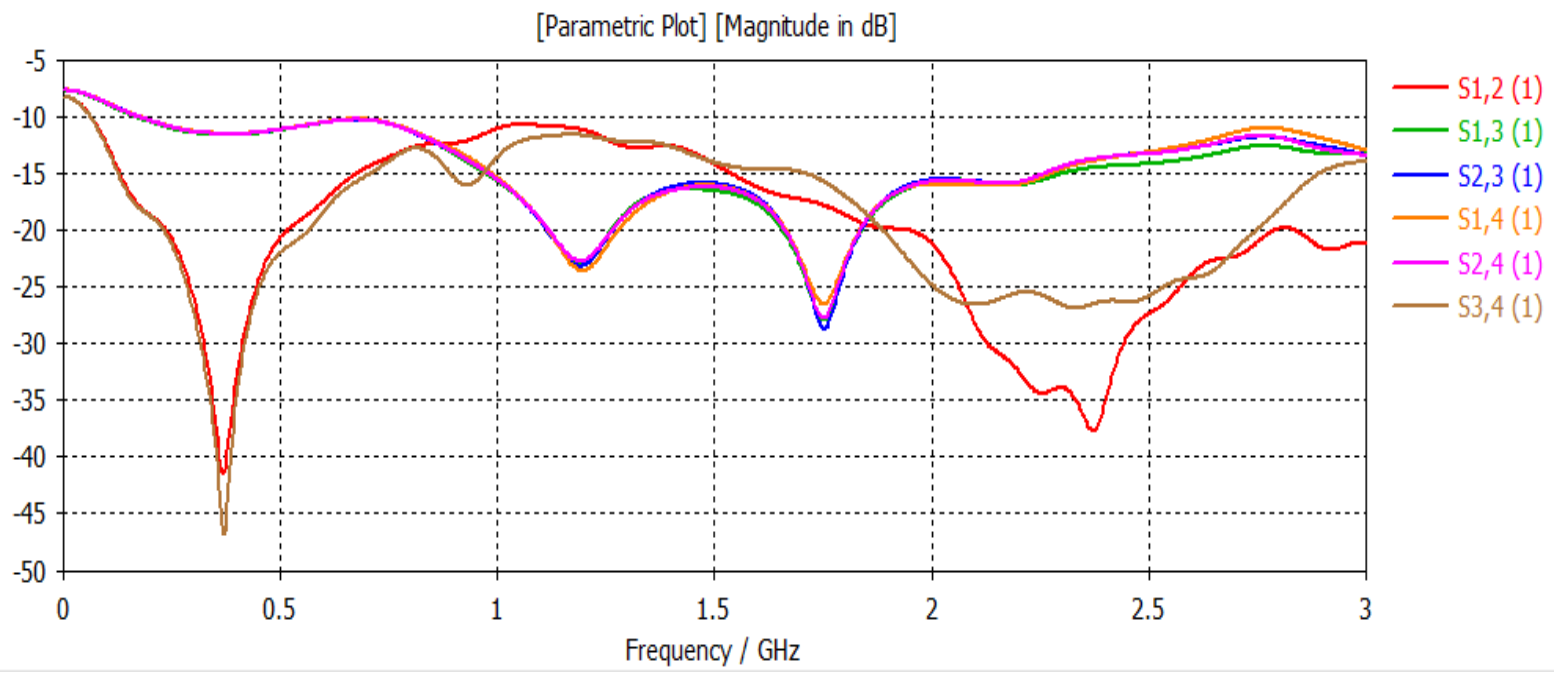

Figure 11: Simulated isolation of the MIMO antenna system

The acceptable value of envelope correlation coefficient (ECC) should be less than 0.5 to provide good diversity performance. The plots of ECC between the ports of the proposed MIMO antenna through far-field are shown in Fig.12. At desired frequency band from 0.85 to $2.6 \mathrm{GHz}$, an ECC value of less than 0.1 is achieved which is sufficient for MIMO applications. The plots of simulated diversity gain of proposed MIMO antenna with slots in the ground plane are shown in Fig.13. The values of the diversity gain about 10dB within the entire desired bandwidth have been achieved.

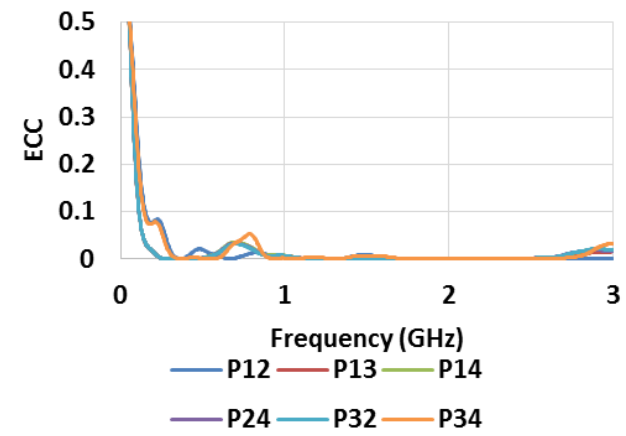

Figure 12:Simulated ECC of the MIMO antenna system 


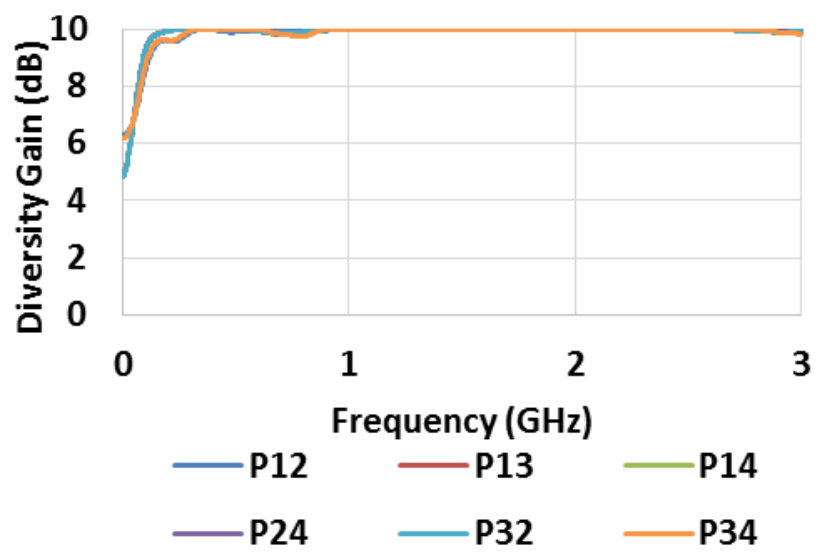

Figure 13: Simulated diversity gain of the MIMO antenna system

A comparison of simulated 2-D radiation patterns of the MIMO antenna system at 0.9 and $2.6 \mathrm{GHz}$ are shown in Fig.14. Due to the symmetric structure of MIMO antenna system, the radiation patterns are plotted for Port 1 and Port 3 only are shown because it is same with the result for Port 2 and 4 respectively. A dual-polarized MIMO antenna has been achieved because of the ports are orthogonal. Therefore, the horizontal and vertical electric fields are produced from Port 1 and 3 respectively. Fig.14 (a) and (b) show a bidirectional E-plane radiation pattern and almost omnidirectional pattern H-plane while (c) and (d) show a nearly omnidirectional radiation pattern without any deep nulls is obtained at the expected operating frequencies.

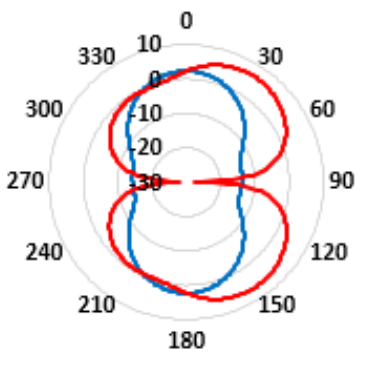

(a)

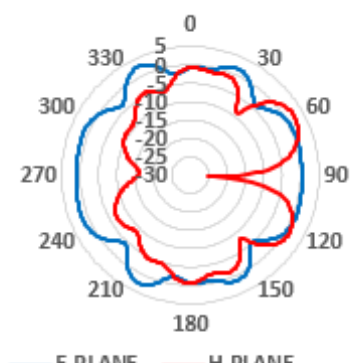

(c)

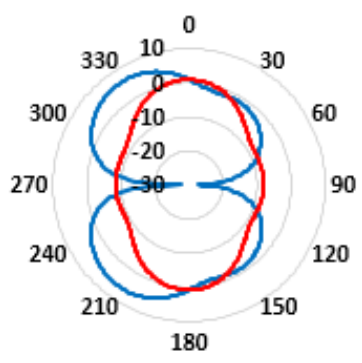

-E-PLANE - H-PLANE

(b)

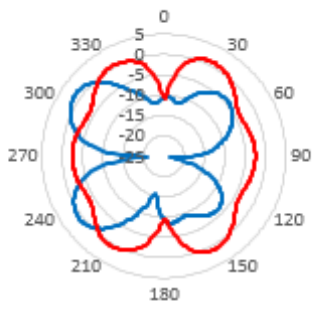

- E-PLANE -H-PLANE

(d)

Figure 14: Simulated radiation pattern of the MIMO antenna system. (a) E-plane and H-plane of port 1 at $0.9 \mathrm{GHz}$, (b) E-plane and $\mathrm{H}$-plane of port 3 at $0.9 \mathrm{GHz}$, (c) E-plane and $\mathrm{H}$-plane of port 1 at 2.6GHz, (d) E-plane and $\mathrm{H}$-plane of port 3 at $2.6 \mathrm{GHz}$.

Fig. 15 and 16 show the simulated maximum gain and efficiencies of the proposed MIMO antenna for the four ports when one port is excited and other ports are terminated. It can be seen that the coverage frequency of simulated results is $0.8-3.0 \mathrm{GHz}$, which provides simulated gains of $4-7 \mathrm{dBi}$ and efficiencies of $60-94 \%$. 


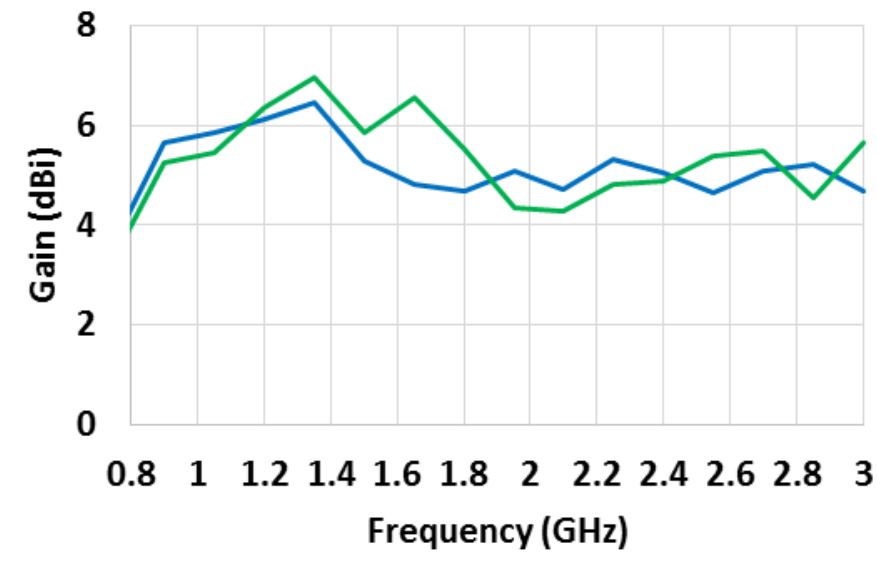

-PORT 1 - PORT 3

Figure 15 : Simulated maximum gain of MIMO antenna system

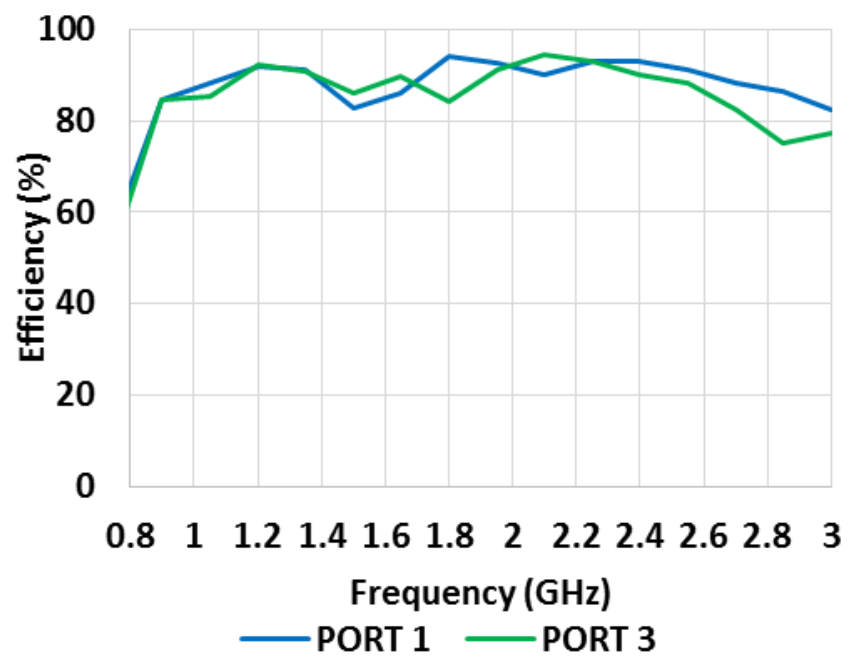

Figure 16 : Simulated efficiency of MIMO antenna system

\section{Conclusion}

The structure of a single port antenna and wideband MIMO antenna system with common elements made up of four microstrip feedline monopole antennas are presented in this paper. The simulated results for single port antenna and MIMO antenna show that the antenna can operate at the desired operating bandwidth. For wideband MIMO antenna system, the simulated results show that the four ports provide $63 \%$ efficiency and a return loss of at least $-10 \mathrm{~dB}$ between 0.85 to $2.6 \mathrm{GHz}$. Therefore, the performance of the proposed MIMO antenna is sufficient to cover the frequency range of $0.85-2.6 \mathrm{GHz}$. Besides, it was verified that a ground plane slots structure can reduce the mutual coupling between the elements. Consequently, the S-parameters in terms of isolation better than -10dB are achieved at the expected frequency bands. The simulated ECC in term of far field less than 0.1 and simulated diversity gains of about $10 \mathrm{~dB}$ are obtained to evaluate the diversity performance of the proposed antenna. The covered frequency band according to the results of S-parameters, radiation characteristics and diversity performance is suitable for GSM, UMTS Wi-Fi and LTE for SCADA wireless communication backhaul application.

\section{Acknowledgement}

The authors would like to express their gratitude to Ministry of Higher Education (MOHE) in Malaysia and Universiti Teknologi Malaysia (UTM) for providing the financial support for this research through the HICoe Research Grant Scheme (R. J130000.7851.4J412) and Universitas Sriwijaya (UNSRI) for sponsoring the Matching Grant Research between UTM and UNSRI. The Grants are.managed by Research Management Centre (RMC) at UTM 


\section{References}

Asset Management Department, TNB “ Handbook of Remote Terminal Unit for Distribution SCADA”, May 2014

Ms Groun Mejda, “ Multiband Microstrip Patch Antenna for 4G (LTE)” Chapter II. Mohamed Khider University Biskra, Faculty of Science and Technology, Department of Electrical Engineering, June 2018

A. Moradikorordalivand, T. A. Rahman, and M. Khalily, "Common Elemnts Wideband MIMO Antenna System for Wi-Fi/LTE Access Point Applications” IEEE Antennas and Wireless Propagation Letters, Vol. 13, 1601 1604,2014

A. Moradikorordalivand and T. A. Rahman, "Broadband Modified Rectangular Microstrip Patch Antenna using Stepped Cut at Four Corners Method”, Progress In Electromagnetic Research, Vol. 137, 599-619, 2013

Malaysian Communications and Multimedia Commision (MCMC), Public Inquiry,"Allocation of spectrum bands for mobile broadband service in Malaysia", July 2019

Alireza Moradi, Razali Ngah, and Mohsen Khalily, "Polarized Diversity Compact Planar MIMO Antenna for Wireless Zccess Point Applications”, Progress In Electromagnetic Research C, Vol. 91, 115-127, 2019

Abubeker A. Yusuf and Selcuk Paker, "Design of Wideband MIMO Antenna for Wireless Applications" 2017 25th Signal Processing and Communications Applications Conference (SIU), Antalya, 2017, pp. 1-4.

A. Moradikorordalivand, T. A. Rahman, Chee Yen Leow and Sepideh Ibrahim, "Dual polarized MIMO antenna system for $\mathrm{WiFi}$ and LTE wireless access point applications", International Journal of Communication Systems , December 2014 